\title{
Ghost-Pulse Reduction in 40-Gb/s Systems Using Line Coding
}

\author{
Bane Vasic, Senior Member, IEEE, Varsha S. Rao, Ivan B. Djordjevic, Raymond K. Kostuk, Member, IEEE, and \\ Ildar Gabitov
}

\begin{abstract}
At high bit rates, four-wave mixing and nonlinearities in the optical channel give rise to ghost pulses. In this letter, we propose the application of modulation codes to tackle this effect.
\end{abstract}

Index Terms-Four-wave mixing (FWM), ghost pulses, modulation codes, optical communications.

\section{INTRODUCTION}

$\mathbf{I}^{1}$ T IS widely accepted that at high bit rates of $40 \mathrm{~Gb} / \mathrm{s}$ or beyond, data transmission through optical channels deployed using dispersion managed schemes is limited by nonlinear effects [1], especially by four-wave mixing (FWM). These effects cause timing and amplitude jitter as well as interactions between the pulses in the channel causing energy transfer between them. Ghost pulses (or shadow pulses) result from this energy transfer. Ghost pulses are formed from interactions between pulses in varied positions, but it has been seen that the presence of pulse triples in what are called "resonance" positions cause heavy energy transfer [2]. If this energy is transferred to a period where no pulse exists (a " 0 " bit period), a ghost pulse is said to have been formed. Over a number of spans, these "ghost" pulses gain sufficient energy to be detected as " $1 \mathrm{~s}$ " by the detector at the output of the channel, thus causing reduction in bit-error-rate (BER) characteristics. A common method used to tackle the problem of suppressing ghost pulses is to find a good modulation format. As the creation of ghost pulses is a phase sensitive effect, these solutions aim to reduce ghost pulses by removing phase coherence in the pulses, emitted by the optical transmitter, in a given neighborhood. In another proposed format [3], the phase of local pairs of " $1 \mathrm{~s}$ " that surround a " 0 " are made to be opposite.

In this work, we propose an alternative method for reducing the occurrence of ghost pulses based on line coding. It can be shown that any modulation format may be viewed as a trivial line code with a memory of one and rate one (see [4] for more details). By using a modulation code, we can work around the limitations of the channel without significantly increasing the complexity of the system. The code we propose may be viewed as a generalized modulation format, in which

Manuscript received February 25, 2004; revised March 27, 2004. This work was supported by the National Science Foundation (NSF) under Grant ITR 0325979.

B. Vasic, V. S. Rao, I. B. Djordjevic, and R. K. Kostuk are with the Department of Electrical and Computer Engineering, University of Arizona, Tucson, AZ 85721-0104 USA (e-mail: vasic@ece.arizona.edu; vrao@ece.arizona.edu; ivan@ece.arizona.edu; kostuk@ece.arizona.edu).

I. Gabitov is with the Department of Mathematics, University of Arizona, Tucson, AZ 85721-0089 USA (e-mail: gabitov@ math.arizona.edu).

Digital Object Identifier 10.1109/LPT.2004.828844 the channel symbol is determined by several previous symbols. The power of this approach results from an increased flexibility in forbidding bit patterns that cause a ghost-pulse effect. A redundancy is added to the input data sequence to help decode the transmitted data correctly. The line code used thus reduces the occurrence of ghost pulses by avoiding bit patterns that cause the effect.

The channel model used for simulations is a realistic one. In order to concentrate on FWM, the noise generated by the various devices was excluded. We take care of modulation, extinction ratio, realistic models (except for noise) of transmitter, optical filter and electrical filter, intersymbol interference, crosstalk effects, Kerr nonlinearities (self-phase modulation, cross-phase modulation, FWM), and dispersion effects (chromatic dispersion, second-order dispersion). For light propagation through the fiber, the nonlinear Schrödinger equation (NLSE) [5] was solved using the split-step Fourier method.

\section{GhOST Pulses}

At bit rates above $40 \mathrm{~Gb} / \mathrm{s}$, nonlinearities cause FWM [1] due to the dependence of the refractive index of the medium on the intensity of the applied electrical field. Intrachannel FWM has been identified as the major nonlinear effect that limits transmission distance in quasilinear fiber-optic communication systems.

To counter dispersion in optical fibers, dispersion-managed schemes are deployed. These setups consist of alternating spans of fibers having positive and negative chromatic dispersion with the value of residual dispersion being low or zero. Thus, the pulses that travel through these systems undergo alternate widening and compression, causing interaction between the pulses when they overlap. These interactions along with nonlinearities present lead to energy transfers that cause the ghost-pulse phenomena.

It has been observed that the most severe problems are caused by pulse triples at positions $k, l$, and $m$, where $k=l+m$ [2]. Unless there is phase cancellation, this represents the resonance condition and creates a ghost pulse at position "zero." Avoiding all such triples is not realistic. But, it has been seen that triples that lie close to each other cause the most problems. One path to take would be to avoid having streams of " $1 \mathrm{~s}$ " that could bleed off a close by solitary "1."

In Fig. 1, we see a case (thick lines) where there is one zero with one pulse in its vicinity on the left and five pulses on the right. Triples of pulses $(3,-1,2),(4,-1,3),(5,-1,4),(6,-1,5)$ are all in resonance. Thus, in the resulting energy transfer [Fig. 1(dotted lines)] to pulse Position 0, the pulse at Position "- 1 " loses a 


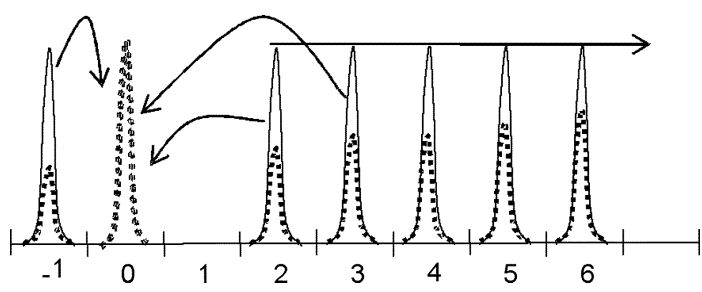

Fig. 1. Ghost created due to pulses in resonance positions.

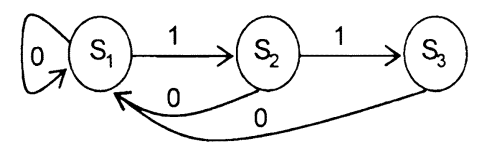

Fig. 2. Directed graph model of our modulation code.

large amount of power since it is involved in four triples. The figure shows an ideal case in the sense no other pulses partake in triples, and effects of other nonlinearities are not shown.

\section{CONSTRAINED SYSTEMS}

In Section II, we have discussed certain bit patterns that cause extensive FWM giving rise to ghost pulses. The optical system can, therefore, be viewed as a constrained system [4].

The role of a line code (also called modulation codes or constrained codes [4]) is to impose certain constraints on a transmitted sequence in order to avoid those waveforms that are most likely to be incorrectly received. A modulation encoder translates an arbitrary user bit stream into a bit stream that satisfies the constraints of the channel. For the price of a slight reduction in user bandwidth and increase in encoder complexity (compared to no modulation), the channel BER is improved as the code works around the constraint that was causing errors in the transmitted bits.

The bipolar alternate mark inversion (AMI) format where alternate " $1 \mathrm{~s}$ " are transmitted with opposite polarity may be considered as a modulation code. The return-to-zero (RZ) modulation format (of duty cycle 0.5 ) can also be considered a modulation code where input " $0 \mathrm{~s}$ " are transmitted at " 00 " and input " $1 \mathrm{~s}$ " transmitted as "01" (within the same pulse interval). The previously mentioned modulation formats with phase variations [2], [3] are also essentially modulation codes. Depending on the type of code, the transmitted bit pattern could depend on one or more previous input bit patterns in addition to the present input. This is seen in the AMI modulation format as well as the proposed phase modulated modulation formats.

As previously mentioned, triples of pulses interact to cause ghost pulses. The closer the triples are in time, the stronger their interaction. The code we use is designed to avoid three or more consecutive "1s." By doing so, we avoid the worst of the triples. Our code construction is based on the constrained system theory from [4] (the rest of this section uses the terminology from [4]).

A directed graph model of the "no more than two adjacent ones" constraint is shown in Fig. 2. Valid sequences can be obtained by reading off the edge labels while making transitions from one state to another according to orientation of the edges.

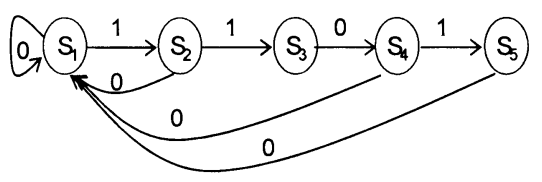

Fig. 3. Directed graph model of modulation code that completely avoids the pattern "11011."

The adjacency matrix for the directed graph shown in Fig. 2 is given by

$$
A=\left[\begin{array}{lll}
1 & 1 & 0 \\
1 & 0 & 1 \\
1 & 0 & 0
\end{array}\right]
$$

Being in a certain state is a representation of what bits have already been generated. For example, state $S_{3}$ is reached only after two consecutive " 1 " bits. Since our constraint is to limit the number of consecutive " 1 " bits to a maximum of two, the next bit has to be a " 0 ." This bit takes us back to state $S_{0}$ from which either " 0 " or " 1 " can be generated.

Since the constraint prevents us from using all possible bit sequences, the capacity of a constrained system, which defines the highest code rate possible, will be less than " 1 ." In fact, it can be shown that the capacity is given by $C=\log _{2} \lambda_{0}$, where $\lambda_{0}$ is the largest eigen value of the adjacency matrix.

Using the largest eigen value of Matrix A, capacity is calculated to be $C=0.8791$. This means that a code with data rates up to 0.8791 can be designed for this constrained system.

On taking the tenth power of the adjacency Matrix A, we see that the largest number on the main diagonal is 274 . Since we need only 256 codewords that are of length 10 bits, this condition guaranties the existence of a $(8,10)$ block code that satisfies the given constraint and has a code rate of 0.8 . Therefore, eight user bits are transmitted as ten encoded bits. The efficiency of this code is given by rate/capacity $=91 \%$.

Further, we choose the 256 code words in such a way as to avoid the pattern "11011" which has two resonance patterns. Since we wanted to design a block code with a reasonable data rate, this pattern could not be completely avoided but its occurrence was kept at a minimum.

By taking higher powers of the graph in Fig. 3, it is possible to design a code where the pattern " 11011 " is completely avoided [for example, a $(16,20)$ block code designed by taking the 20th power of the graph), but this means the lookup table for the block code will be very large. So all things considered, the (8, 10) block code we have used is a good choice.

The encoder can be designed as a finite state machine (FSM) by taking the tenth power of the directed graph, or as a lookup table. The FSM approach is usually not preferred because of timing errors and error propagation. The decoder can be designed as a "sliding window" machine that takes into account some $m$ previous and $n$ following code words. In such a system, error propagation is limited to $n+m+1$ code words. In other words, a wrongly detected code word will only affect the detection of a few of its neighbors, not the entire bit stream (catastrophic) as would be the case in state dependent decoders.

\section{RESULTS}

To study FWM, we use a noiseless dispersion-managed $40-\mathrm{Gb} / \mathrm{s}$ single-channel system. The optical channel is sim- 


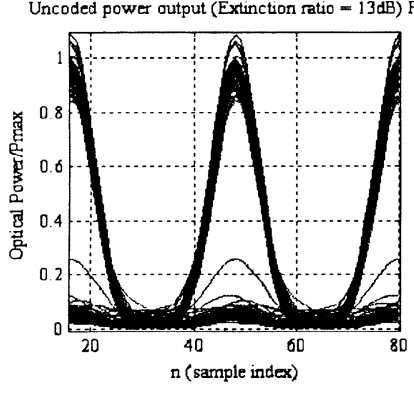

(a)

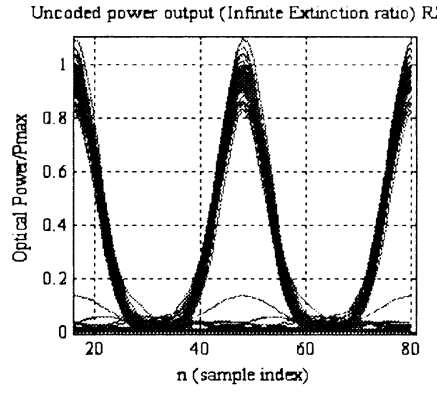

Fig. 4. Eye diagrams (32 samples per bit) after $1200 \mathrm{~km}$ for average powers of $0 \mathrm{dBm}$ with a precompensation of $-320 \mathrm{ps} / \mathrm{nm}$ and zero residual dispersion. (a) Uncoded eye with extinction ratio of $13 \mathrm{~dB}$. (b) Uncoded eye with infinite extinction ratio.

ulated by solving the NLSE [5] using the split-step Fourier method. The dispersion map [6] is of length $L=48 \mathrm{~km}$ and consists of $2 L / 3 \mathrm{~km}$ of $\mathrm{D}^{+}$fiber followed by $L / 3 \mathrm{~km}$ of $\mathrm{D}^{-}$ fiber. The fiber parameters are as follows. $\mathrm{D}^{+}$fiber: dispersion of $20 \mathrm{ps} /(\mathrm{nm} \cdot \mathrm{km})$, dispersion slope of $0.06 \mathrm{ps} /\left(\mathrm{nm}^{2} \cdot \mathrm{km}\right)$, effective area equal to $110 \mu \mathrm{m}^{2}$, and loss equal to $0.19 \mathrm{~dB} / \mathrm{km}$. $\mathrm{D}^{-}$fiber: dispersion of $-40 \mathrm{ps} /(\mathrm{nm} \cdot \mathrm{km})$, dispersion slope of $-0.12 \mathrm{ps} /\left(\mathrm{nm}^{2} \cdot \mathrm{km}\right)$, effective area equal to $30 \mu \mathrm{m}^{2}$, and loss equal to $0.25 \mathrm{~dB} / \mathrm{km}$. The average dispersion and dispersion slope of the map are zero. The nonlinear Kerr coefficient is $2.6 \times 10^{-20} \mathrm{~m}^{2} / \mathrm{W}$. Linear precompensation and postcompensation are also used. 25 spans of this map are used to form a channel that is $1200 \mathrm{~km}$ long. The simulations were carried out with an average channel power of $0 \mathrm{dBm}$ with a central wavelength of $1552.54 \mathrm{~nm}$. A 1024-bit-long pseudorandom binary sequence was used to generate the uncoded eye. This same sequence was encoded and sent over the channel to generate the encoded eyes. To overcome loss, amplifiers (erbium-doped fiber amplifier) are used after every section of fiber, but amplified spontaneous emission noise was ignored. (The aim of this letter is to show our code's capability to reduce the ghost-pulse effect.)

Fig. 4 shows the eye diagram observed at receiver input when the uncoded bit stream is transmitted through the system. Two cases are considered. In the first case, an extinction ratio of $13 \mathrm{~dB}$, typical for Mach-Zehnder modulators available at $40 \mathrm{~Gb} / \mathrm{s}$, is assumed [Fig. 4(a)], while in the second case the extinction ratio is assumed to be infinite [Fig. 4(b)].

Fig. 5 shows the eye diagrams observed for the encoded bit stream. In the first case [Fig. 5(a)], the encoded system increases the $Q$ factor by $4.56 \mathrm{~dB}$. In the second case [Fig. 5(b)], the $Q$ factor is increased by $6.80 \mathrm{~dB}$. [The $Q$ factor is calculated after optical filtering (of bandwidth $80 \mathrm{GHz}$ ), photodetection and electrical filtering (of bandwidth $26 \mathrm{GHz}$ ).] $Q$ factor improvement is defined as $20 \log \left(Q_{\text {encoded }} / Q_{\text {uncoded }}\right)$. In addition to reducing the occurrence of ghost pulses, the modulation code also improves immunity to intrachannel cross-phase modulation.

The simulation results presented here are in excellent agreement with VPItransmissionMaker WDM version 5.5. Fig. 6 illustrates the dependence of $Q$ factor improvement on the number of spans of the map. It is appropriate to mention at this point that our simulation results indicate that the gain in

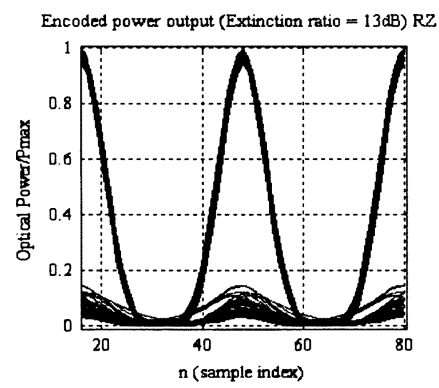

(a)

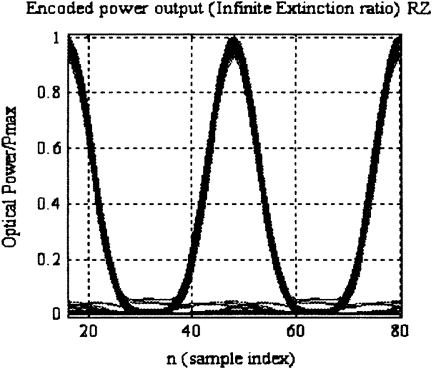

(b)
Fig. 5. (a) Encoded eye with extinction ratio of 13 dB. (b) Encoded eye with infinite extinction ratio.

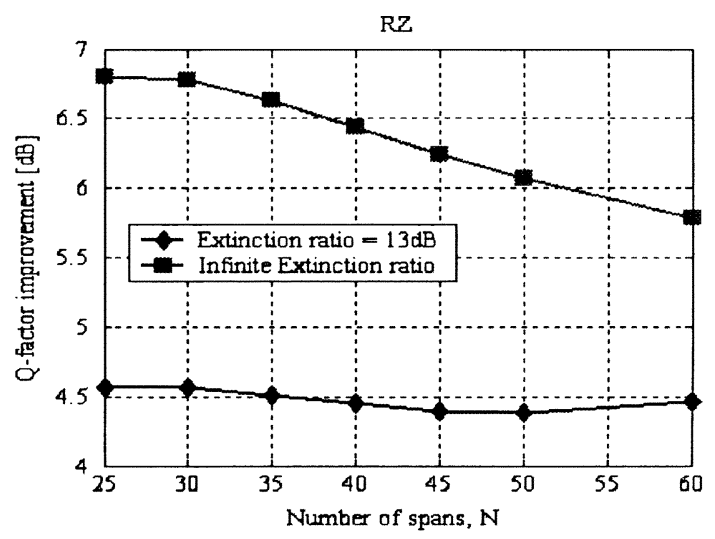

Fig. 6. $Q$ factor improvement for different number of spans.

$Q$ can be further increased by replacing RZ modulation with AMI modulation. The detailed analysis of such a scheme is beyond the scope of this letter, and will, therefore, be discussed elsewhere.

\section{CONCLUSION}

Recently, several different approaches have been proposed in order to reduce the ghost-pulse effect [2], [3]. To tackle this effect, we proposed a rather different approach. Instead of inventing novel modulation formats, we propose the use of modulation codes. Our results show that it is possible to successfully tackle the detrimental effects of FWM in 40-Gb/s systems using simple modulation coding techniques.

\section{REFERENCES}

[1] R. J. Essiambre, B. Mikkelson, and G. Raybon, "Intra-channel crossphase modulation and four-wave mixing in high-speed TDM systems," Electron. Lett., vol. 35, pp. 1576-1578, 1999.

[2] M. Forzati et al., "Reduction of intrachannel four-wave mixing using the alternate-phase RZ modulation format," IEEE Photon. Technol. Lett., vol. 14, pp. 1285-1287, Sept. 2002.

[3] X. Liu et al., "Suppression of interchannel four-wave-mixing-induced ghost pulses in high-speed transmissions by phase inversion between adjacent marker blocks," Opt. Lett., vol. 27, pp. 1177-1179, July 2002.

[4] D. Lind and B. Marcus, Symbolic Dynamics and Coding, Cambridge, U.K.: Cambridge Univ. Press, 1995.

[5] G. P. Agrawal, Nonlinear Fiber Optics. San Diego, CA: Academic, 2001.

[6] J. Zweck and C. R. Menyuk, "Analysis of four-wave mixing between pulses in high-data-rate quasilinear subchannel-multiplexed systems," Opt. Lett., vol. 27, pp. 1235-1237, July 2002. 